\title{
El Control Ambiental en los Archivos como Medio para la Conservación
}

\author{
$M^{a}$ del Pilar Toro Sánchez-Blanco \\ Asesora técnica de Conservación e Investigación \\ del Archivo Histórico Provincial de Sevilla
}

L conservación es el conjunto de operaciones que tiene como objeto prolongar la vida de un ente material, merced a la previsión del daño o a la corrección del deterioro.

La finalidad en el campo de los archivos es mantener las propiedades físicas y culturales de aquellos documentos que han alcanzado la categoría de tales.

Por ello este tipo de conservación se fundamenta en el principio de que todo documento posee una naturaleza corpórea o física, un soporte y unos elementos sustentados que albergan el mensaje privativo del documento. Por tanto la conservación tiene que atender tanto a la integridad física del mismo como a la funcional; es decir, debe mantener la permanencia, que hace referencia a la conservación de la naturaleza física, y la durabilidad, a la capacidad de transmitir información, de la pieza en cuestión.

Para conservar la materia existen dos vías de actuación: la prevención del deterioro (preservación) y la reparación del daño (restauración).

La preservación en archivos, así como en toda institución que custodie bienes culturales, va encaminada a eliminar el daño ocasionable por factores ambientales o fortuitos, que se ciernen en el medio que rodea el bien a conservar; por tanto los métodos y medios preventivos no suelen ser de aplicación directa, sino que se dirigen al ambiente para controlar las condiciones microclimáticas, con objeto de erradicar los agentes nocivos o los elementos que temporal o permanentemente pueden influir en la degradación.

Los criterios de preservación advierten de la necesidad de crear un medioambiente acorde a las exigencias de permanencia y durabilidad. Este principio implica el conocimiento previo del comportamiento físico y químico de la estructura y elementos del bien a conservar, y las causas potenciales de su deterioro, teniendo en cuenta la variedad, tamaño y formato de soportes y elementos sustentados que pueden encontrarse en los archivos: papel, pergamino, cristal, celuloide, soportes magnéticos y ópticos y tintas caligráficas, de impresión o pictóricas, emulsiones fotosensibles, sellos pendientes de metal o cera, de placa...
Los métodos preventivos van dirigidos de esta manera a frenar, en lo posible, el proceso degradatorio. Así, la preservación es tarea prioritaria en la conservación, pues aunque se restaure es necesaria para evitar futuros daños.

Atendiendo al origen de las causas de degradación se puede hablar de extrínsecas (localizadas en el medio y acondicionamiento de los documentos) e intrínsecas (derivadas de los materiales que los conforman y estructuran).

Las causas extrínsecas son ajenas al documento y la mayoría de ellas son totalmente previsibles al ser consustanciales al microclima que rodea el documento. Hacia estas causas llamadas naturales van dirigidas preferentemente los procedimientos preventivos, porque las alteraciones de origen intrínseco son fomentadas no pocas veces, por la agresividad del ambiente.

Dentro de las causas naturales son de gran importancia las alteraciones provocadas por el binomio temperaturahumedad relativa.

Cuando la humedad alcanza valores extremos, puede llegar a degradar los materiales orgánicos, favorecer la corrosión de los metales y la proliferación de organismos biodegradadores (bacterias, hongos, pececillos de plata y termes). La temperatura, en estrecha relación con la humedad, actúa acelerando o retardando la actividad biológica y las reacciones químicas; la ventilación si es incontrolada, puede alterar los niveles adecuados de humedad relativa y temperatura del aire, permitir la entrada de contaminantes gaseosos, polvo, hollín, esporas e insectos que favorecerían procesos corrosivos, manchas y proliferación de plagas sobre los documentos; y si excede ciertos niveles de velocidad, 0 '3 m/seg., puede producir depósitos de suciedad.

Las características de construcción del edificio (tipo de aislamiento de los muros; proporción y distribución de vanos y paramentos..) que produzcan focos de humedad y acúmulos de suciedad pueden influir en los anteriores factores, disminuyendo la efectividad de las medidas de control de las condiciones ambientales que dispongamos.

Por otro lado la actividad humana intervendrá positiva o negativamente en el mantenimiento de un ambiente "sano" para la documentación.

Sin embargo no podemos olvidar que todos estos factores están interrelacionados y que la modificación de uno solo de ellos afectará al resto, alterándolos en mayor o menor grado. Por tanto, antes de actuar hemos 
de valorar el sistema complejo formado por los documentos como bien cultural a conservar, el edificio como continente que los alberga y los factores que los condicionan y afectan.

Por ello, y para poder establecer un plan coherente de medidas de conservación, es necesario conocer la construcción y equipamientos técnicos de nuestro edificio, pues constituirán las medidas pasivas de prevención; aunque por muy buenas y adecuadas que sean nunca podremos asegurar la protección total de los documentos y siempre será necesario recurrir a medidas activas de lucha contra los factores de degradación. de los factores ambientales, pues se manifiesta en aquellas zonas de un paramento interior en zonas oscuras y mal ventiladas, cuya temperatura superficial es más fría que la interior del edificio y que el valor estimado como punto de rocío.

El emplazamiento de los depósitos dentro del edificio también influirá en las condiciones ambientales de cada uno de ellos, así como las medidas preventivas contra incendios, robos y actos vandálicos estarán en función de la adecuada distribución de los circuitos de la documentación y del público en general.

El análisis del edificio debe comenzar por sus condicionantes externos, fundamentalmente el clima de la zona y la ubicación y el entorno del mismo.

Dependiendo de las características del clima local, los depósitos deben tener la orientación que mejor tienda a mantener la estabilidad en el grado de humedad relativa y temperatura adecuados a la conservación de los fondos, así como la cantidad de luz que pueden recibir. El edificio tiene que ser como una membrana protectora de los cambios climáticos, pero interesará saber hasta qué punto los amortigua; por ello influirá, además de las características constructivas, la orientación del mismo con respecto a la luz solar y a los vientos.

El tipo de suelo (flojo o deslizable, húmedo, seco y polvoriento, infectados o propensos a la contaminación biológica, inundable...), sobre el que se asiente el edificio de archivos, exige medidas constructivas que corrijan los aspectos que puedan resultar perjudiciales para la conservación.

La proximidad al centro cultural, y la calidad de las redes de comunicación, determinarán la facilidad de acceso para investigadores y público en general, así como el índice de contaminación atmosférica, acústica, biológica y el nivel de vibraciones. Ser un edificio exento, rodeado por otras construcciones o adosado a ellas, así como si es de nueva planta o adaptado, influirá notablemente en las medidas preventivas de protección y barrera.

En cuanto a los condicionantes internos que pueden modificar el microclima del edificio debemos tener en cuenta las características constructivas y arquitectónicas del edificio en general.

El cerramiento exterior tiene que asegurar la estanqueidad y el aislamiento de la humedad y la temperatura, de manera que se mantenga la inercia térmica del interior del edificio amortiguando los saltos de temperatura de la noche al día. Si se produce un problema de humedad en los elementos constructivos tenemos que identificar el origen de la misma, es decir, si procede de filtración, capilaridad o condensación. En principio todas son consecuencia del defecto de equipamiento en los mismos; sin embargo, la condensación depende más de la conjunción

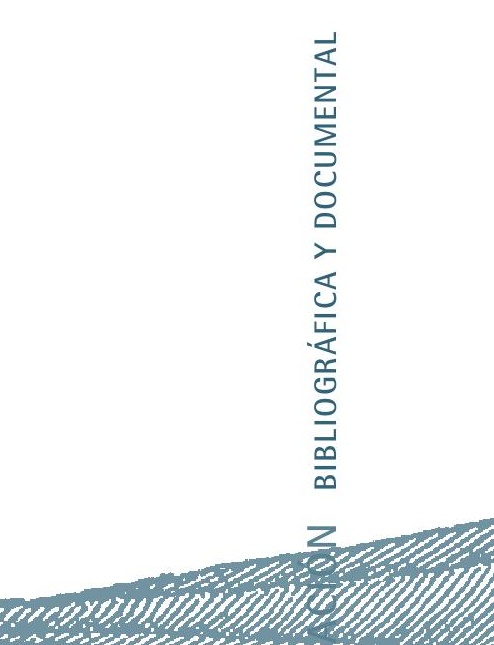

estudio de

los depósitos, inde-

pendientemente de su ubicación, debemos analizar el mobiliario que contienen, ya que su distribución dentro de él, así como el material del que esté construido, reaccionarán de distinta manera a unas mismas condiciones ambientales. El mobiliario más habitual lo constituyen las estanterías fijas o móviles y los planeros; estos últimos para la instalación en horizontal de los documentos que, por contener sellos pendientes, por su formato o por el estado de conservación así lo requieran.

Los criterios de preservación advierten de la necesidad de crear un medioambiente acorde a las exigencias de permanencia y durabilidad. Este principio implica el conocimiento previo del comportamiento físico y químico de la estructura y elementos del bien a conservar

Las estanterías de madera

son buenas reguladoras de la temperatura y humedad relativa; sin embargo deben tener un tratamiento ignífugo y antitermitas. Las metálicas por el contrario pueden sufrir condensación de humedad ante un descenso brusco de la temperatura.

Por otra parte, las estanterías fijas, sean de madera o metal, no deben estar adosadas a la pared y tener la balda inferior separada del suelo al menos $6 \mathrm{cms}$. De esta manera facilitamos la limpieza del suelo y evitamos la acumulación 
de polvo y suciedad, agentes favorables para la proliferación de insectos. Por otra parte impedimos la condensación de la humedad en los paramentos y su contagio a la documentación, pues destruye los puentes de hidrógeno de la celulosa, foco de atracción para las termes. Además estos insectos lucífugos son capaces de crear unos conductos sobre la pared o las estanterías que los facultan para llegar hasta dicho alimento protegidos de la luz.

Una vez consideradas a priori todas las circunstancias que pueden afectar a la estabilidad de la temperatura y la humedad relativa en los depósitos debemos proceder al control de ambos factores.

Partimos de la base de la necesidad de conocer cuáles son las condiciones ideales de conservación para cada tipo de soporte, pero aún más es saber si en nuestros depósitos se cumplen.

Es frecuente encontrar en la bibliografía especializada unas condiciones ambientales óptimas para la conservación de los distintos soportes tales como: $18-20^{\circ} \mathrm{C}$ de temperatura y $45-60 \%$ de humedad relativa para el papel; entre $35-58 \%$ HR para el pergamino y entre 25-35\% para fotografías en color.

Sabemos que una humedad relativa alta unida a una temperatura alta propicia el desarrollo de hongos, bacterias e insectos tanto en papel como en negativos fotográficos; que una humedad relativa baja y temperatura alta, resquebraja y abarquilla los pergaminos, vuelve frágil al papel y cuartea los negativos.

Esto demuestra la importancia de la relación entre temperatura y humedad relativa como factor de estabilización o por el contrario, de degradación en la conservación de los documentos. Sin embargo lo que la mayoría de las veces desconocemos es que más perjudicial que sobrepasar los parámetros aconsejables para cada soporte, son las oscilaciones tanto diarias como estacionales, y especialmente los cambios bruscos y rápidos de uno o ambos factores. Cuestión a tener muy en cuenta en aquellos depósitos climatizados artificialmente $\mathrm{o}$ en las salidas de documentos para exposiciones temporales o bien simplemente a la sala de lectura.

En caso de préstamo de documentos, las condiciones microclimáticas que deben exigirse durante el transporte y en la exposición a la que vayan destinados, no serán las óptimas que hemos expresado más arriba, sino precisamente aquellas a la que están habituados.

El control de las condiciones ambientales adecuadas nos exige conocer realmente los niveles de estos factores y sus oscilaciones durante un período mínimo anual. Para ello necesitaremos un equipo básico compuesto por termohigrógrafos para la medición continua y registro de la temperatura y humedad relativa dispuestos en cada depósito, en la sala de lectura y en la de exposiciones, si la hubiere, y un termohigrómetro electrónico para medidas puntuales y para tomarlo como referencia para calibrar los anteriores aparatos.
Los termohigrógrafos más habituales en nuestros archivos recogen en una gráfica durante siete días la temperatura y humedad relativa, siendo conveniente cambiar la gráfica cada semana el mismo día y a la misma hora. La colocación dentro del depósito debe ser a una altura media sobre la pared, entre 0.80 y $1.50 \mathrm{~m}$ del suelo, evitando la proximidad de puertas, ventanas, conductos de aireación o salidas de aire acondicionado. La calibración de este aparato debe hacerse cada tres meses para obtener lecturas fiables y puede hacerse comparando con un termohigrómetro electrónico, como ya hemos indicado.

Una vez instalado el equipo obtendremos las medidas de temperatura y humedad relativa de nuestros depósitos a través de las gráficas, pero para poder controlar las condiciones ambientales debemos analizar dichas medidas. Para ello es conveniente diseñar unos impresos de recogida de datos teniendo en cuenta el tipo de aparatos y los datos que suministran.

Los modelos que presentamos como ejemplo han sido diseñados en el Archivo Histórico Provincial de Sevilla y son el resultado de la evolución producida según las necesidades y la experiencia obtenida a lo largo de más de un año.

Son tres los impresos de recogida de datos: de control semanal de cada depósito, el resumen de datos semanales de todos los depósitos y el de control mensual.

\section{En este modelo se presentan dos cuadros.}

En el primero, en la columna vertical de la izquierda (fecha) debe rellenarse el día concreto del mes y año a que corresponde cada día de la semana. Cada día a su vez está subdividido en dos líneas, una para los valores obtenidos de la humedad relativa y otra para los de la temperatura. Los casilleros numerados de 2 a 24 se refieren a las horas del día y se corresponden con las mismas franjas horarias de las gráficas de los termohigrógrafos, de manera que los datos reflejados son las medidas correspondientes a las intersecciones del registro continuo cada dos horas.

En las dos columnas finales se dispondrán las medias aritméticas diarias de la humedad relativa y de la temperatura; y la oscilación diaria de ambas, obtenida de restar a la máxima la mínima.

El sombreado de las casillas correspondientes a las horas entre las 8 y las 14, de lunes a viernes, obedece al interés en saber hasta qué punto afecta al depósito el permanecer abierto durante la jornada laboral o si por el contrario no existe influencia alguna, pues hemos podido comprobar que durante esas horas en días de trabajo los depósitos pueden llegar a sufrir importantes y bruscas oscilaciones de humedad relativa, mientras que la temperatura no muestra variación apenas. Durante los días no laborables estos depósitos mantienen la temperatura y humedad relativa constantes; es decir, que cuando se mantienen cerrado se produce la inercia térmica y la estanqueidad a la que antes habíamos hecho mención respecto al edificio. 


\begin{tabular}{l}
\begin{tabular}{|l}
\hline \multicolumn{1}{|c|}{ CONTROL SEMANAL DE HUMEDAD RELATIVA Y TEMPERATURA } \\
ARCHIVO HISTORICO PROVINCIAL DE SEVILLA
\end{tabular} \\
\hline Depósito \\
\hline FECHA
\end{tabular}

DATOS SEMANALES. Medias de :

$\mathrm{HR}$

Oscilación

Temperatura

Oscilación

OBSERVACIONES

\begin{tabular}{|c|c|c|c|c|c|c|c|c|c|c|c|c|c|c|c|c|}
\hline \multicolumn{17}{|c|}{$\begin{array}{c}\text { RESÚMEN DE DATOS SEMANALES DE LOS DEPÓSITOS } \\
\text { ARCHIVO HISTÓRICO PROVINCIAL DE SEVILLA }\end{array}$} \\
\hline \multirow[t]{2}{*}{ Fecha } & \multicolumn{4}{|l|}{$\mathrm{Cl}$} & \multicolumn{4}{|l|}{$\mathrm{BI}$} & \multicolumn{4}{|l|}{$C 2$} & \multicolumn{4}{|l|}{ B2 } \\
\hline & $\overline{\mathrm{HR}}$ & OSC & $\bar{T}$ & OSC & $\mathrm{HR}$ & OSC & $\mathrm{T}$ & OSC & $\mathrm{HR}$ & OSC & $\bar{T}$ & OSC & $\mathrm{HR}$ & OSC & $\mathrm{T}$ & OSC \\
\hline & & & & & & & & & & & & & & & & \\
\hline & & & & & & & & & & & & & & & & \\
\hline & & & & & & & & & & & & & & & & \\
\hline & & & & & & & & & & & & & & & & \\
\hline & & & & & & & & & & & & & & & & \\
\hline & & & & & & & & & & & & & & & & \\
\hline & & & & & & & & & & & & & & & & \\
\hline & & & & & & & & & & & & & & & & \\
\hline & & & & & & & & & & & & & & & & \\
\hline & & & & & & & & & & & & & & & & \\
\hline & & & & & & & & & & & & & & & & \\
\hline & & & & & & & & & & & & & & & & \\
\hline & & & & & & & & & & & & & & & & \\
\hline & & & & & & & & & & & & & & & & \\
\hline & & & & & & & & & & & & & & & & \\
\hline
\end{tabular}

OBSERVACIONES 


\begin{tabular}{|c|c|c|c|c|c|c|c|c|c|c|c|c|c|c|c|c|}
\hline MES & & $\begin{array}{l}\mathrm{C} \\
\mathrm{AN}\end{array}$ & NTRC & L M & $\begin{array}{l}\text { NSUA } \\
\text { RCHIV }\end{array}$ & $\begin{array}{l}\text { L D } \\
\text { D HIS }\end{array}$ & $\begin{array}{l}\text { HUM } \\
\text { ÓRICC }\end{array}$ & $\begin{array}{l}\text { EDA } \\
\text { PRC }\end{array}$ & $\begin{array}{l}\text { REL } \\
\text { INCIA }\end{array}$ & $\begin{array}{l}\text { ATIV } \\
-D E\end{array}$ & $\begin{array}{c}\text { Y TE } \\
\text { VILLA }\end{array}$ & MPE & ATU & & & \\
\hline & $\mathrm{Cl}$ & & & & $\mathrm{BI}$ & & & & C2 & & & & B2 & & & \\
\hline & $\mathrm{HR}$ & & $\mathrm{T}$ & & $H R$ & & $\mathrm{~T}$ & & $H R$ & & $\mathrm{~T}$ & & $H R$ & & $\mathrm{~T}$ & \\
\hline & \begin{tabular}{|l|} 
Med. \\
Diaria \\
\end{tabular} & Osc & $\begin{array}{l}\text { Med. } \\
\text { Diaria }\end{array}$ & Osc & $\begin{array}{l}\text { Med. } \\
\text { Diaria }\end{array}$ & Osc & \begin{tabular}{|l} 
Med. \\
Diaria
\end{tabular} & Osc & $\begin{array}{l}\text { Med. } \\
\text { Diaria }\end{array}$ & Osc & $\begin{array}{l}\text { Med. } \\
\text { Diaria }\end{array}$ & Osc & $\begin{array}{l}\text { Med. } \\
\text { Diaria }\end{array}$ & Osc & $\begin{array}{l}\text { Med. } \\
\text { Diaria }\end{array}$ & Osc \\
\hline 1 & & & & & & & & & & & & & & & & \\
\hline 2 & & & & & & & & & & & & & & & & \\
\hline 3 & & & & & & & & & & & & & & & & \\
\hline 4 & & & & & & & & & & & & & & & & \\
\hline 5 & & & & & & & & & & & & & & & & \\
\hline 6 & & & & & & & & & & & & & & & & \\
\hline 7 & & & & & & & & & & & & & & & & \\
\hline 8 & & & & & & & & & & & & & & & & \\
\hline 9 & & & & & & & & & & & & & & & & \\
\hline 10 & & & & & & & & & & & & & & & & \\
\hline 11 & & & & & & & & & & & & & & & & \\
\hline 12 & & & & & & & & & & & & & & & & \\
\hline 13 & & & & & & & & & & & & & & & & \\
\hline 14 & & & & & & & & & & & & & & & & \\
\hline 15 & & & & & & & & & & & & & & & & \\
\hline 16 & & & & & & & & & & & & & & & & \\
\hline 17 & & & & & & & & & & & & & & & & \\
\hline 18 & & & & & & & & & & & & & & & & \\
\hline 19 & & & & & & & & & & & & & & & & \\
\hline 20 & & & & & & & & & & & & & & & & \\
\hline 21 & & & & & & & & & & & & & & & & \\
\hline 22 & & & & & & & & & & & & & & & & \\
\hline 23 & & & & & & & & & & & & & & & & \\
\hline 24 & & & & & & & & & & & & & & & & \\
\hline 25 & & & & & & & & & & & & & & & & \\
\hline 26 & & & & & & & & & & & & & & & & \\
\hline 27 & & & & & & & & & & & & & & & & \\
\hline 28 & & & & & & & & & & & & & & & & \\
\hline 29 & & & & & & & & & & & & & & & & \\
\hline 30 & & & & & & & & & & & & & & & & \\
\hline 31 & & & & & & & & & & & & & & & & \\
\hline $\begin{array}{l}\text { Med. } \\
\text { mens. }\end{array}$ & & & & & & & & & & & & & & & & \\
\hline OBS & $\mathrm{Cl}$ & & & & & & & & & & & & & & & \\
\hline
\end{tabular}

El segundo cuadro se ha dejado para resumen de los datos semanales obtenidos de la media aritmética de los diarios (humedad relativa y su oscilación; temperatura y su oscilación) y para las observaciones, en donde tiene cabida todo aquello que pueda ser de interés o que creamos que pueda influir en las condiciones ambientales: días de lluvia, tiempo atmosférico aproximado, fecha de limpieza del depósito, funcionamiento o desconexión de ventiladores, humidificadores, deshumidificadores..., medidas que se establecen para mejorar dichas condiciones; o bien sobre el propio termohigrógrafo, fecha de calibración, diferencias de grados y porcentaje al ser calibrado...
El segundo impreso recoge el resumen de los datos semanales de todos los depósitos. La primera columna, la de fecha, debe expresar las extremas de lunes a domingo de cada semana.

Este modelo ha sido diseñado para comparar las medidas de cuatro depósitos. La distribución dentro del edificio puede ser de interés a la hora de agruparlos dentro de cada impreso.

Dentro de cada semana y depósito pondremos los datos medios semanales tomados del segundo cuadro del 
impreso anterior. Se han calculado quince semanas como promedio de las que ocupa cada estación anual; de tal manera que en cuatro de estos modelos podemos tener reflejado un año entero.

Por último, en el recuadro de las observaciones deben reflejarse todas aquellas incidencias surgidas al comparar unos depósitos con otros y que puedan estar relacionadas con la situación dentro del edificio, la orientación, la altura... En el Archivo Histórico Provincial de Sevilla los depósitos con termohigrógrafos, que son ocho, están distribuidos en cuatro plantas y en ellos la temperatura es mayor conforme vamos ascendiendo en altura; dos depósitos además están en una entreplanta sin ventilación directa al exterior, por lo que las oscilaciones diarias de humedad relativa son menores que en los restantes. Este modelo ofrece la posibilidad, por tanto, de comparar las condiciones de los depósitos, desde ligeras variaciones a cruciales diferencias; a nosotros corresponde valorarlas e identificar en lo posible las causas.

Un último modelo establece la recogida de datos de todo el mes en cada depósito. Estos se disponen en la primera columna de la izquierda ( $\mathrm{Cl}$ al B2) y los datos que se reflejarán serán las medias aritméticas diarias de la temperatura y humedad relativa y las oscilaciones correspondientes de ambos factores. Estos datos proceden de las dos últimas columnas de la derecha del primer modelo.

En la columna de medias mensuales se obtendrá la media aritmética de: la humedad relativa media diaria, la oscilación diaria, la temperatura media diaria y la oscilación diaria de la misma por depósitos. En las observaciones haremos igual que en los modelos anteriores: valorar los datos, señalar cualquier anomalía y sus posibles causas, y reseñar las medidas tomadas en caso de ser necesario intervenir sobre las condiciones ambientales de uno o más depósitos.

La cumplimentación de estos modelos nos permite conocer de manera objetiva cuáles son las condiciones ambientales que se mantienen a lo largo del año en cada depósito, las oscilaciones diarias y estacionales y sobre todo si es necesario influir de manera directa sobre ellas para corregirlas, cuándo y por cuánto tiempo o si por el contrario estamos desestabilizando unas adecuadas condiciones naturales.
Para concluir, debemos considerar que para poder conservar adecuadamente el patrimonio documental a nuestro cargo, los profesionales de archivo necesitamos contar con medios técnicos suficientes y con una mayor formación e información en el campo de la conservación preventiva..

Bibliografía

DUCHEIN, Micheil. El edificio como medio de protección de los archivos. UIMP. Sevilla, 1995.

HERRÁEZ, Juan Antonio; RODRíGUEZ LORITE, Miguel Ángel. Manual para el uso de aparatos y toma de datos de las condiciones ambientales en museos. Ministerio de Cultura. Madrid, 1989.

KRAEMER KOELLER, Gustav. Tratado de la previsión del papel y de la conservación de bibliotecas y archivos. Tomo II. Madrid, 1993.

PESCADOR DEL HOYO, Ma del Carmen. El archivo. Instalaciones y conservación. Ed. Norma. Madrid, 1988.

VALENTÍN, Nieves. Contaminación microbiológica en materiales de archivo y bibliotecas. Técnicas de evaluación y sistemas de control. UIMP. Sevilla, 1995.

VIÑAS, Vicente; VIÑAS, Ruth. Las técnicas tradicionales de restauración. Un estudio del RAMP. Programa general de Información y UNISIST. Organización de las Naciones Unidas para la Educación, la Ciencia y la Cultura. París, 1988.
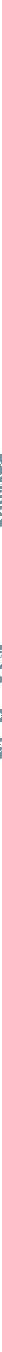